\title{
Development and Validation of a RP-HPLC Method for the Estimation of Amlexanox in Oral Paste Dosage Form
}

\author{
Abdullah Al Masud¹, Mohammad Saydur Rahman², Towfika Islam, Saki Sultana, \\ Moynul Hasan ${ }^{1}$ and A. B. M. Faroque ${ }^{1}$ \\ ${ }^{1}$ Department of Pharmaceutical Technology, Faculty of Pharmacy, University of Dhaka, \\ Dhaka-1000, Bangladesh \\ ${ }^{2}$ Department of Pharmacy, Jagannath University, Dhaka-1100, Bangladesh
}

\begin{abstract}
A simple, reproducible and efficient reversed phase high performance liquid chromatographic (RPHPLC) method has been developed for the estimation of a recently approved anti allergic drug, amlexanox in oral paste dosage form. The separations were carried out on a Zorbax Eclipse XBD, $\mathrm{C}_{18}$ column $(150 \mathrm{x} 4.6 \mathrm{~mm}$; $5 \mu \mathrm{m})$ at a flow rate of $1.50 \mathrm{ml} / \mathrm{min}$. by using mobile phase comprising of mixed buffer (pH adjusted to 6.50) and methanol (50:50 v/v). The injection volume was $10 \mu \mathrm{l}$ and the peaks were detected at $244 \mathrm{~nm}$. The linear dynamic range found to be in the concentration range of $15-35 \mu \mathrm{g} / \mathrm{ml}$ and coefficient of correlation was found to be 0.999 . The \%RSD value was below 2.0 for intra-day and inter-day precision which indicated that the method was highly precise. The LOD (Limit of detection) and LOQ (Limit of quantitation) were found to be $3.8 \mathrm{ng} / \mathrm{ml}$ and $12.5 \mathrm{ng} / \mathrm{ml}$, respectively which revealed that the method was highly sensitive. The percentage recovery of amlexanox ranged from 99.31 to $99.75 \%$, indicating the accuracy of the method and absence of interference from the excipients present in the formulation. The proposed method was simple, fast, accurate and reproducible and hence can be applied for routine quality control operations of amlexanox in oral paste dosage form.
\end{abstract}

Key words: Amlexanox, Anti allergic, RP-HPLC, LOD, LOQ.

\section{INTRODUCTION}

Amlexanox is an antiallergic drug, clinically effective for atopic diseases, especially allergic asthma and rhinitis. Amlexanox, as a topical paste, is a well tolerated treatment of recurrent apthous ulcers. Recurrent apthous ulcer (RAU) is the most prevalent oral mucosal disease in humans, estimated to affect between $5 \%$ and $50 \%$ of the general population. ${ }^{1}$ Chemically, amlexanox is 2-amino-7-isopropyl-5oxo-5H-[1]benzopyrano[2,3-b] pyridine-3-carboxylic acid. It has a molecular formula of $\mathrm{C}_{16} \mathrm{H}_{14} \mathrm{~N}_{2} \mathrm{O}_{4}$ and has a molecular weight of 298.30. Amlexanox is odorless, white to yellowish-white crystalline powder. $^{2}$

Correspondence to: A.B.M. Faroque

Tel: +8801830002826, Fax: 9674273

E-mail: abmfaroque@yahoo.com

Dhaka Univ. J. Pharm. Sci. 10(2): 67-70, 2011 (December)
The structural formula is shown below:<smiles>CC(C)c1ccc2oc3nc(N)c(C(=O)O)cc3c(=O)c2c1</smiles>

Amlexanox

Amlexanox is a mucoadhesive oral paste which has been clinically proven to abort the onset, accelerate healing and resolve pain of apthous ulcers. It decreases the durath of healing and thereby decreases the pain sensation. Recent studies have also shown that the majority of ulcers can be prevented by application of the paste during the prodromal (preulcerative) phase of the disease. Recurrent Apthous Ulcers (RAU), also known as Recurrent Apthous Stomatitis (RAS), is recognized as the most common oral mucosal disease known to man and about 20- 
$25 \%$ of the general populations suffer at least one incidence of apthous ulcers each year. Amlexanox is also being investigated for its anti-allergic and antiinflammatory properties. ${ }^{3-14}$

A literature survey revealed that very few analytical methods have been reported for the determination of amlexanox in oral paste preparation $^{16-17}$ and some of those methods are laborious and not reproducible at all. So attempts have been made to develop an accurate, precise and economically viable reversed phase HPLC method for the estimation of amlexanox content in oral paste preparation.

\section{MATERIALS AND METHODS}

Chemicals and reagents. Amlexanox INN was obtained from "ACI Pharmaceuticals Ltd." Narayanganj, Bangladesh, as a gift. Analytical grade monobasic sodium phosphate dihydrate (Scharlau, Spain), 65\% ethanol (Scharlau, Spain), triethylamine (Scharlau, Spain), imidazole (Merck, Germany), $\mathrm{NaOH}$ (Scharlau, Spain), HCl (Scharlau, Spain), HPLC grade methanol (Scharlau, Spain), and distilled water were used. The buffer was prepared by dissolving $6.5 \mathrm{~g}$ of monobasic sodium phosphate dihydrate in $900 \mathrm{ml}$ of distilled water and subsequent addition of $1.0 \mathrm{ml}$ of triethylamine and the $\mathrm{pH}$ was adjusted to 6.50 with $2 \mathrm{M} \mathrm{NaOH}$ solution. Imidazole solution was prepared by dissolving $8.25 \mathrm{~g}$ of imidazole in $100 \mathrm{ml}$ of distilled water and the $\mathrm{pH}$ was adjusted to 6.75 to 6.85 by addition of $37 \%$ hydrochloric acid. The commercially available amlexanox oral paste claimed to contain 5\% w/w (50 mg of amlexanox per gram of paste) were procured from local market.

Apparatus and chromatographic condition. The chromatographic separation was performed on a prominence Shimadzu (Japan) high performance liquid chromatographic instrument equipped with a Zorbax Eclipse XBD, $\mathrm{C}_{18}$ column (150 x 4.6 mm; 5 $\mu \mathrm{m})$ integrated with PDA (Photo Diode Array) detector where detection was carried out at $244 \mathrm{~nm}$. The mobile phase consisting of the buffer ( $\mathrm{pH}$ 6.50) and methanol at a ratio of 50:50 (v/v) was freshly prepared, filtered and sonicated before use and delivered at a flow rate of $1.50 \mathrm{ml} / \mathrm{min}$. Ethanol $65 \%$ was used as diluting medium. The volume of each injection was $10 \mu \mathrm{l}$. The column and the HPLC system were kept in ambient temperature.

Preparation of stock solution. Stock solution of amlexanox was prepared by dissolving $50.0 \mathrm{mg}$ of amlexanox in $200 \mathrm{ml}$ of volumetric flask containing $10 \mathrm{ml}$ of imidazole solution and the solution was sonicated for 5 minutes and made up to the mark with $65 \%$ ethanol to get a concentration of $0.25 \mathrm{mg} / \mathrm{ml}$. Subsequent dilutions of this solution were made with $65 \%$ ethanol to get concentrations of $15-35 \mu \mathrm{g} / \mathrm{ml}$. The standard solution prepared as mentioned above was injected into the loop and the chromatogram was recorded.

Treatment of sample (oral paste). An accurately weighed portion of oral paste equivalent to $25.0 \mathrm{mg}$ of amlexanox was taken into a $100 \mathrm{ml}$ volumetric flask, $10 \mathrm{ml}$ of imidazole solution was added to it and shaken well. Then the volume was adjusted up to the mark with $65 \%$ ethanol and sonicated for 5 minutes. It was filtered through Whatman filter paper-01. The solution thus obtained was diluted with $65 \%$ ethanol so as to obtain a concentration range of linearity as previously discussed for pure drug. Sample solution was injected under the chromatographic conditions as mentioned above and the chromatogram was recorded.

\section{RESULTS AND DISCUSSION}

All of the analytical parameters for the proposed method were determined according to the International Conference on Harmonization (ICH) guidelines.

Linearity. The linearity of the method was determined at five concentration levels from 15-35 $\mu \mathrm{g} / \mathrm{ml}$. The plot of peak area of each sample against respective concentration of amlexanox was found to be linear in the range of $15-35 \mu \mathrm{g} / \mathrm{ml}$. Beers law was found to be obeyed over this concentration range. The regression equation was found to be $\mathrm{Y}=52349 \mathrm{x}$ - 61950 and the correlation coefficient (r) of the standard curve was 0.999 . 
Table 1. Validation parameters.

\begin{tabular}{lc}
\hline Parameters & \multicolumn{1}{c}{ Results } \\
\hline Linearity range $(\mu \mathrm{g} / \mathrm{ml})$ & $15-35$ \\
Standard regression equation & $\mathrm{Y}=52349 \mathrm{x}-61950$ \\
Correlation coefficient & 0.999 \\
LOD $(\mathrm{ng} / \mathrm{ml})$ & 3.8 \\
LOQ $(\mathrm{ng} / \mathrm{ml})$ & 12.5 \\
\hline
\end{tabular}

Precision. The precision is a measure of the ability of a method to generate reproducible results. The precision of the assay was determined by repeatability (intra-day) and intermediate precision (inter-day) and reported as \%RSD. For repeatability, six determinations of $100 \%$ test concentration (25 $\mu \mathrm{g} / \mathrm{ml}$ ) were measured and the $\%$ RSD was calculated. For intermediate precision the same work was done in three consecutive days and average \%RSD value was calculated. The intra-day and inter-day precision measurements showed (Table 2.) good reproducibility with percent relative standard deviation (\%RSD) values below $2 \%$. This indicated that method was highly precise.

Table 2. Intra-day and inter-day precision of amlexanox $(n=6)$

\begin{tabular}{cccc}
\hline $\begin{array}{l}\text { Concen- } \\
\text { tration } \\
(\mu \mathrm{g} / \mathrm{ml})\end{array}$ & $\begin{array}{c}\text { Concentration } \\
\text { found }(\mu \mathrm{g} / \mathrm{ml}) \\
(\text { Mean } \pm \text { S.D. })\end{array}$ & \% RSD & $\begin{array}{c}\text { \% RSD } \\
\text { (Average) }\end{array}$ \\
\hline $\begin{array}{c}\text { Intra-day } \\
25\end{array}$ & $24.93 \pm 0.04$ & 0.160 & 0.160 \\
$\begin{array}{c}\text { Inter-day } \\
25\end{array}$ & $24.88 \pm 0.05$ & 0.200 & \\
25 & $24.90 \pm 0.03$ & 0.120 & 0.147 \\
25 & $24.72 \pm 0.03$ & 0.121 & \\
\hline
\end{tabular}

Recovery studies (Accuracy). Recovery studies were performed to judge the accuracy of the method. The studies were carried out by adding a known quantity of pure drug to the pre-analyzed formulation and the proposed method was followed. Formulation concentration was $25 \mu \mathrm{g} / \mathrm{ml}$ at three levels of drug addition such as $60 \%, 100 \%$ and $140 \%$. Six replicates of each concentration was measured. The percentage recovery values (Table 3), which were near about $100 \%$, indicated the accuracy of the method and absence of interference from the excipients present in the formulation.

Sensitivity. Limit of detection (LOD) and limit of quantitation (LOQ) were determined at signal-tonoise ratio of 3:1 and 10:1 respectively. The LOD and LOQ for amlexanox were found to be $3.8 \mathrm{ng} / \mathrm{ml}$ and $12.5 \mathrm{ng} / \mathrm{ml}$, respectively (Table 1.) which demonstrated that the method was highly precise.

Table 3. Accuracy of the method for the determination of amlexanox $(n=6)$

\begin{tabular}{ccccc}
\hline $\begin{array}{c}\text { Level of } \\
\text { addition } \\
(\%)\end{array}$ & $\begin{array}{c}\text { Formu- } \\
\text { lation } \\
(\mu \mathrm{g} / \mathrm{ml})\end{array}$ & $\begin{array}{c}\text { Addition } \\
\text { of pure } \\
\text { drug } \\
(\mu \mathrm{g} / \mathrm{ml})\end{array}$ & $\begin{array}{c}\text { Total amount of } \\
\text { drug recovered } \\
(\mu \mathrm{g} / \mathrm{ml})\end{array}$ & $\begin{array}{c}\text { Recovery } \\
(\text { Mean } \pm \text { S.D. })\end{array}$ \\
$\begin{array}{ccccc}(\text { Mean } \pm \\
\text { S.D. })\end{array}$ \\
\hline 60 & 25 & 15 & $39.90 \pm 0.05$ & $99.75 \pm 0.12$ \\
100 & 25 & 25 & $49.70 \pm 0.15$ & $99.39 \pm 0.30$ \\
140 & 25 & 35 & $59.58 \pm 0.26$ & $99.31 \pm 0.44$ \\
\hline
\end{tabular}

Selectivity and stability. For these studies, standard, sample and placebo preparations, all in both normal and stressed condition (at $60{ }^{\circ} \mathrm{C}$ for 24 hours) were studied. But in both normal and stressed conditions, no significant change in peak area, due to degeneration or any other way was found which revealed the stability of the analyte (amlexanox). On the other hand, in both conditions, no interference of excipients or degradation products (Figure 1) was found in the peak region of amlexanox, indicating the selectivity and specificity of the method.

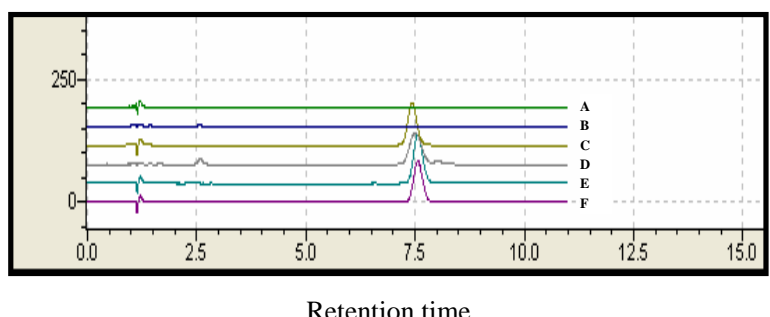

Figure 1. Representative chromatograms obtained from A) Placebo preparation (normal condition) B) Placebo preparation (stressed condition) C) Standard preparation (normal condition) D) Sample preparation (normal condition) E) Sample preparation (normal condition) F) Sample preparation (stressed condition).

System suitability test. The system suitability tests were carried out on freshly prepared standard stock solution of amlexanox to evaluate the suitability of the system as presented in Table 4 . From the typical chromatogram of amlexanox as shown in Figure 1, it was found that the average retention time \pm standard deviation for amlexanox found to be $7.47 \pm 0.035$ minute for six replicate injections. The asymmetry factor was found to be 1.09, which indicated symmetric nature of the 
column. The number of theoretical plates was found to be 5601, which suggested an efficient performance of the column. The absence of additional peaks in the chromatogram indicated non-interference by the common excipients used in the oral paste. To optimize the chromatographic conditions, various combination of buffer and methanol were tested and the ratio of 50:50 v/v afforded peak with good shape and resolution.

Table 4. System suitability parameters

\begin{tabular}{lc}
\hline Retention time (min.) \pm S.D. & $7.47 \pm 0.035$ \\
\hline No. of theoretical plates & 5601 \\
Asymmetric factor & 1.09 \\
\hline
\end{tabular}

Robustness. Robustness was performed by small but deliberate variation in the chromatographic conditions and was found to be unaffected by small variations like $\pm 2 \%$ in volume of mobile phase composition, $\pm 1 \%$ change in column temperature. It was observed that there were no marked changes in the chromatograms, which demonstrated the method was robust.

Assay of amlexanox. The method also applied for the assay of Amlexanox in oral paste formulation (in triplicate) and the results are shown below in Table 5 . The results obtained were in good agreement with the claimed potency.

Table 5. Determination of active ingredients in oral paste preparation.

\begin{tabular}{lccc}
\hline Sample & $\begin{array}{c}\text { Label } \\
\text { claim }\end{array}$ & $\begin{array}{c}\text { Amount found } \\
\text { (Avg.) }\end{array}$ & $\begin{array}{c}\% \text { amount } \\
\text { found }\end{array}$ \\
\hline $\begin{array}{l}\text { Amlexanox } \\
\text { oral paste }\end{array}$ & $5 \%(\mathrm{w} / \mathrm{w})$ & $4.94(\% \mathrm{w} / \mathrm{w})$ & 98.80 \\
\hline
\end{tabular}

\section{CONCLUSION}

From the above discussion it is clear that the proposed method was simple, sensitive and reliable with good precision and accuracy. Hence, this method can be used for the routine quality control operations for amlexanox in oral paste dosage form.

\section{ACKNOWLEDGEMENT}

The authors are thankful to "ACI Pharmaceuticals Ltd." for providing necessary facilities to conduct this research work.

\section{REFERENCES}

1. Bell, J. 2005. Amlexanox for the treatment of recurrent apthous ulcers. Clin. Drug Investig. 25, 555-66.

2. http://www.rxlist.com/apthasol-drug.htm.

3. Barrons, R. W. 2001. Treatment strategies for recurrent oral apthous ulcers. Am. J. Health Sys. Pharm. 58, 41-53.

4. Akintoye, S. O., Greenberg, M. S. 2005. Recurrent apthous stomatitis. Dent. Clin. North Am. 49, 31-47.

5. Felice, F., Alessandro, L., Curzio, B., Fernando, G., Monica, N., Silvia, P., Nicola, C. 2007. Guideline for Diagnosis and Management of apthous stomatitis. Pediatr. Infect Dis. J. 26, 728-732.

6. Juliette, B. 2005. Amlexanox for the treatment of recurrent apthous ulcers. Clin. Drug Invest. 25, 555-566.

7. Natah, S. S., Kontinen, Y. T., Enattah, N. S., Assamakhi, N., Sharkey, K.A., K.A., Hayrinenimmonen, R. 2004. Recurrent apthous ulcers today: a review of the growing knowledge. Int. J. Oral Maxillofac. Surg. 33, 221-234.

8. Greer, R. O., Lindenmuth, J. E., Juarez, T., Khandwala, A. 1993. A double-blind study of topically applied 5\% amlexanox in the treatment of apthous ulcers. J. Oral Maxillofac. Surg. 51, 243-248.

9. Khandwala, A., Van Inwegen, R. G., Alfano, M. C. 1997. 5\% amlexanox oral paste, a new treatment for recurrent minor apthous ulcers: I. clinical demonstration of acceleration of healing and resolution of pain, Oral Surg. Oral Pathol. Oral Radiol. Endod. 83, 222-230.

10. Binnie, W. H., Curro, F. A., Khandwala, A., Van Inwegen, R. G. 1997. Amlexanox oral paste: a novel treatment that accelerates the healing of apthous ulcers. Compend. Contin. Educ. Dent. 18, 1116-1118.

11. Murray, B., Biagioni, P. A., Lamey, P. J. 2006. The efficacy of amlexanox oral disc (TM) on the prevention of recurrent minor apthous ulceration. J. Oral Path. Med. 35, 117-122.

12. Rodriguez, M., Rubio, J.A., Sanchez, R. 2007. Effectiveness of two oral pastes for the treatment of recurrent apthous stomatitis. Oral Diseases. 13, 490-494.

13. Saijo, T., Kuriki, H., Ashida, Y. 1985. Mechanism of the action of amlexanox (AA-673), an orally active antiallergic agent. Int. Arch Allergy Appl. Immunol. 78, 43-50.

14. Murray, B., Mcguinness, N., Biagioni, P., Hyland, P., Lamey, P.J. 2005, A comparative study of the efficacy of Aptheal ${ }^{\circledR}$ in the management of recurrent minor apthous ulceration. $J$. Oral Pathol. Med. 34, 413-419.

15. ICH guidelines, analytical method validation (Q3). Geneva, july 2000.

16. Sun, L., Ding, J. and Zhang, D. Z. 2009. RP-HPLC determination of related substances of amlexanox and its content in paste. Yaowu Fenzi Zazhi. 29, 1220-1222.

17. McComish, S., Mize, A., Harris, C., Premcumer, N. and Colon, L.E. 2003. Validation of an HPLC method for the determination of amlexanox in human serum. analytical Biochemistry (ABC) Laboratories, Inc., 7200 E. ABC Lane, Columbia, MO, Access Pharmaceuticals Inc., Dallas, TX. 
